\title{
Review of: "An Archaea-Specific c-type Cytochrome Maturation Machinery is Crucial for Methanogenesis in Methanosarcina acetivorans"
}

\author{
James Ferry ${ }^{1}$ \\ 1 Pennsylvania State University
}

Potential competing interests: The author(s) declared that no potential competing interests exist.

The authors have investigated c-type cytochromes in the model methanogen Methanosarcina acetivorans. They confirm the suspected role of genes in maturation, and with bioinformatic analyses, show the genes are likely acquired by HGT that was also shown for many $M$. acetivorans genes. Importantly, the work supports previous biochemical studies (Ref. 26) showing a role for MmcA in electron transport specific for acetate conversion to methane. However, an important discussion is missing regarding the conflicting report that MmcA is not necessary, also based on a MmcA deletion mutant (Ref. 28). Significant, although not surprising, the work shows that c-types are less essential for growth on methylotrophic substrates. Finally, the following sentence in the significance statement is grossly misleading: "However, neither the biogenesis nor the role of c-type 47 cytochromes in methane metabolism has ever been investigated" (see citations 26 and 28). Overall, the work represents a worthy advancement in characterization of c-type cytochromes in methanogens. 